\title{
On the applicability of hybrid functionals for predicting fundamental properties of metals
}

\author{
Weiwei $\mathrm{Gao}^{1}$, Tesfaye A. Abtew ${ }^{1}$, Tianyi Cai ${ }^{1}$, Y. Y. Sun ${ }^{2}$, S. B. Zhang ${ }^{2}$, and Peihong Zhang ${ }^{1}$ \\ ${ }^{1}$ Department of Physics, University at Buffalo, State University of New York, Buffalo, New York 14260, USA \\ ${ }^{2}$ Department of Physics, Applied Physics and Astronomy, \\ Rensselaer Polytechnic Institute, Troy, New York 12180, USA
}

(Dated: December 18, 2015)

\begin{abstract}
The repercussions of an inaccurate account of electronic states near the Fermi level by hybrid functionals in predicting several important metallic properties are investigated. The difficulties include a vanishing or severely suppressed density of states (DOS) at $E_{F}$, significantly widened valence bandwidth, greatly enhanced electron-phonon (el-ph) deformation potentials, and an overestimate of magnetic moment in transition metals. The erroneously enhanced el-ph coupling calculated by hybrid functionals may lead to a false prediction of lattice instability. The main culprit of the problem comes from the simplistic treatment of the exchange functional rooted in the original Fock exchange energy. The use of a short-ranged Coulomb interaction alleviates some of the drawbacks but the fundamental issues remain unchanged.
\end{abstract}

\section{INTRODUCTION}

As one of the most successful and extensively implemented theories in electronic structure methods, the importance of density functional theory (DFT) $[1,2]$ can not be overstated. However, despite its tremendous triumph in describing many ground state properties, DFT within the local density approximation (LDA) [2, 3] or the generalized gradient approximation (GGA) [4-6] has serious limitations when it comes to describing excited state properties of materials, a problem that is often loosely referred to as the LDA band gap problem.

Strictly speaking, the Kohn-Sham (KS) eigenvalues cannot be interpreted as the quasiparticle energies in solids. Nevertheless, there has been much effort put into the development of better energy functionals that can also provide a more faithful description of the quasiparticle energies. One class of such functionals, known as hybrid functionals [7], in which the conventional LDA or GGA exchange-correlation functional is mixed with a fraction of the Hartree-Fock (HF) exchange energy, has the promises of providing a better description of a wide range of materials properties [8-15], including the band gap of semiconductors. There are notable exceptions, however. For example, the experimental band gap of $\mathrm{FeS}_{2}$, an earth abundant material with a very high optical absorption coefficient in the visible region, is about $0.95 \mathrm{eV}[16,17]$, but the Heyd-Scuseria-Ernzerhof (HSE) $[18,19]$ hybrid functional predicts a band gap of about $2.6 \mathrm{eV}$. [20]

Hybrid functionals have been unbiasedly applied to the study of various materials systems and properties involving metals, and, in some cases, improved or similar results compared with the LDA or GGA functionals have been obtained [12, 21-24]. In this work, however, we point out that hybrid functionals have to be used with great caution for metallic systems. In particular, for metallic properties that are sensitive to (or determined by) the Fermi surface properties (electronic structure near the Fermi energy), hybrid functionals are likely to give significantly worse results compared with the LDA or GGA. We show that the application of hybrid functionals to metallic systems gives rise to a range of false or inaccurate results. These include, but not limited to, 1) enlarged valence bandwidth, 2) vanishing or greatly suppressed density of states (DOS) at the Fermi level, 3) significantly overestimated el-ph deformation potentials, 4) false predictions of lattice instability for systems (e.g., $\mathrm{MgB}_{2}$ ) with a strong el-ph coupling, and 5) enhanced magnetism in transition metals. The occupied band width and the electronic structure near the Fermi leve are among the most important properties of metals. Many fundamental thermal and transport properties of metals are controlled or strongly affected by the electronic structure near the Fermi level. The inaccurate prediction of the strength of the el-ph coupling, lattice stability, and magnetism are just a few manifestations of the fundamental difficulties that are inherent to hybrid functionals when they are applied to metallic systems. We believe that the aforementioned problems are serious enough to justify a thorough re-evaluation of the applicability of hybrid functionals to metals and degenerate semiconductors.

\section{COMPUTATIONAL DETAILS}

Our density functional calculations are performed using the Quantum Espresso [25] package. The GGA functional of Perdew, Burke, and Ernzerhof (PBE) [26] and hybrid functionals, both the PBE0 [27] and the HSE06 [18, 19] functionals, are used in this work. Electron-ion interactions are described by normconserving pseudopotentials [28]. The plane wave basis has an energy cutoff of $20 \mathrm{Ry}$ for the calculations of $\mathrm{Na}$, $50 \mathrm{Ry}$ for $\mathrm{MgB}_{2}$, and $180 \mathrm{Ry}$ for transition metals. For transition metals, all semicore subshells (i.e., $3 s, 3 p$, and 
$3 d)$ are included as valence electrons.

\section{RESULTS AND DISCUSSION}

We first investigate the electronic structure of metals calculated with the PBE0 and HSE06 functionals using $\mathrm{Na}$ as an example. Figure 1 compares the DOS of Na calculated using both the PBE (blue), HSE06 (green), and the PBE0 (red) functionals. The DOS are calculated using a very high $k$-point density of $40 \times 40 \times 40$ with a small gaussian smearing parameter of $0.01 \mathrm{eV}$. It is wellestablished that the PBE functional gives a satisfactory description of the electronic structure for a wide range of materials including metals. In fact, the calculated bandwidth $(3.15 \mathrm{eV})$ of Na agrees very well with that obtained from the free-electron model (shown in Fig. 1 with the black curve superimposed on the PBE result). The PBE (and free-electron model) result also compares reasonably well with experiment $[29,30]$ other than a wellunderstood quasiparticle renormalization factor. [31] The PBE0 functional, in contrast, gives an overly-exaggerated occupied bandwidth of $4.2 \mathrm{eV}$. Perhaps a more alarming issue is that the PBE0 functional predicts a vanishing DOS at $E_{F}$ as shown in Fig. 1. Note that the singularity is not clearly shown in the figure because of a finite smearing parameter used in our calculation. Nevertheless, the significant dip in the DOS at the $E_{F}$ can be clearly seen. The abnormal DOS calculated with the PBE0 functional is a direct result of the HF exchange energy incorporated in the functional. A simple freeelectron calculation using the HF theory [32] (with the HF exchange-energy rescaled by a factor of 0.25 as it is used in the PBE0 functional) gives nearly identical results as shown with the black curve superimposed on the red curve (PBE0 results). The HSE06 functional gives results that are somewhat in between those calculated with PBE and PEB0 functionals. The bandwidth is still significantly over estimated, however. Although there is no singularity in the calculated DOS, the HSE06 functional gives a ssuppressed DOS near the Fermi energy.

The Fermi-surface property is perhaps one single most important property of metals. A wrong description of the Fermi-surface properties, including the DOS and the electron energy dispersion near the Fermi level, will have serious consequences when it comes to predicting other fundamental properties of metals, especially for properties that are sensitive to the Fermi surface properties (electronic structure near the Fermi energy). These properties include, but not limit to, el-ph coupling strength, magnetism, thermal properties such as electronic free energy and specific heat of metals, and transport properties.

In order to demonstrate the repercussion of the incorrect description of the electronic structure of metals near the Fermi level, we investigate some electronphonon coupling related aspects of metals using $\mathrm{MgB}_{2}$,

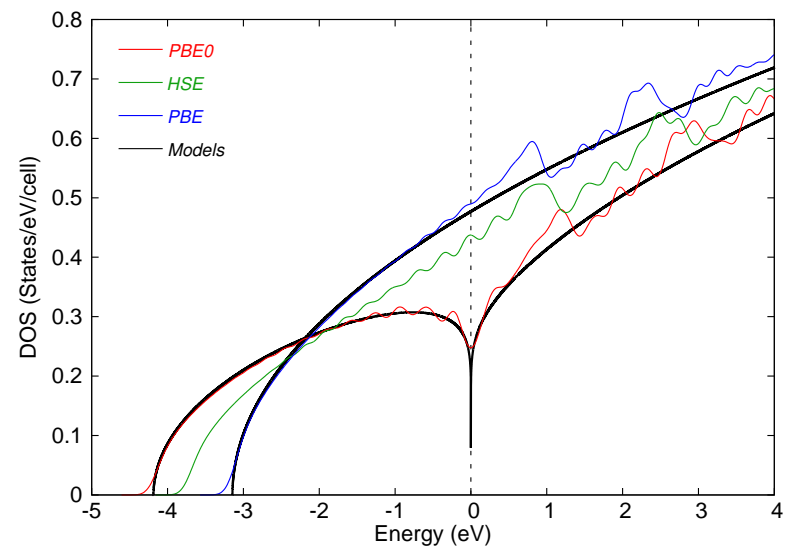

FIG. 1: (color online) Calculated DOS of sodium using the PBE (in blue) HSE06 (green), and PBE0 (red) functionals. The PBE result agrees well with that of the free-electron model (solid black curve), and the PBE0 result can be well understood within the HF theory of free electrons (solid black curve) that shows vanishing DOS at the $E_{F}$ (shown with the dashed line).

a well-established multi-gap phonon-mediated superconductor [33-37], as an example. Quantitative predictions of the superconductivity in $\mathrm{MgB}_{2}$, including the precise calculation of the superconducting transition temperature $T_{C}$, is one of the celebrated [35-37] successes of modern electronic structure methods. We mentioned that all previous calculations [35-39] used either LDA of GGA functionals. This success would not be possible without an accurate description of the electronic structure near $E_{F}$. Figure 2 shows the DOS of $\mathrm{MgB}_{2}$ calculated using the PBE0 (shown in red) and PBE (in blue) functionals. Similar to the case of Na, the PBE0 functional gives a significantly widened occupied bandwidth and a greatly suppressed DOS near $E_{F}$. In fact, a fullyconverged PBE0 calculation (with respect to the $k$-grid density) should give a precisely zero DOS at $E_{F}$.

It is now well-established that the strong coupling between the $E_{2 g}$ phonons (shown in Fig 3) and the boronderived $p \sigma$ states is largely responsible for the enormously high $T_{C}$ of $\mathrm{MgB}_{2}$, and previous work [35-39] has demonstrated the accuracy of LDA or GGA functionals in the prediction of the phonon energies and their coupling with electrons. Figure 4 shows the band structure of $\mathrm{MgB}_{2}$ calculated with boron atoms displaced by 0.05 a.u. from their equilibrium position along one of the $E_{2 g}$ phonon modes shown in Fig. 3. The PBE results are shown with blue solid curves and the PBE0 results are shown with red dashed curves. The degeneracy of the $p \sigma$ states along the $\Gamma \rightarrow A$ direction is lifted by the $E_{2 g}$ phonon distortion. An and Pickett [35] used this energy shift (splitting) to calculate the el-ph deformation potential of the $E_{2 g}$ phonons and successfully estimated the $T_{C}$ to be in the range of $32 \sim 46 \mathrm{~K}$ with GGA. Our calculated $E_{2 g}$ defor- 


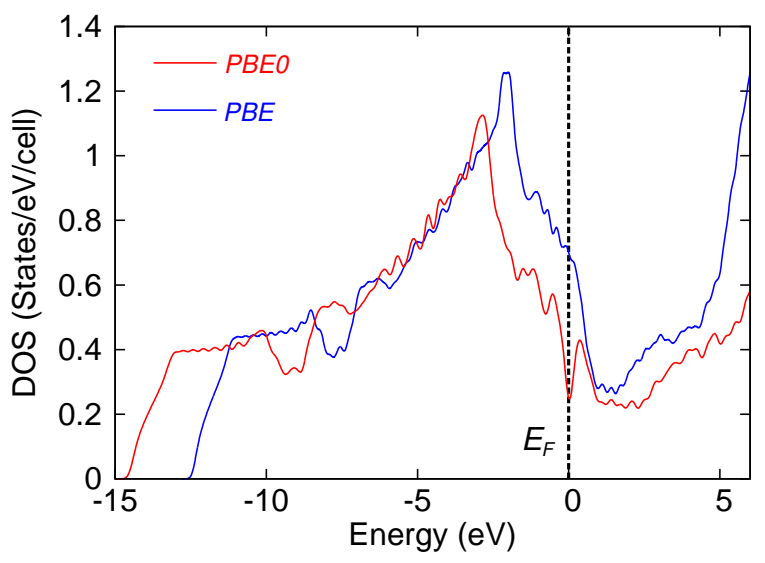

FIG. 2: (color online) Density of states of $\mathrm{MgB}_{2}$ calculated using the PBE (blue) and PBE0 (red) functionals. The dashed line indicates the Fermi level $E_{F}$.

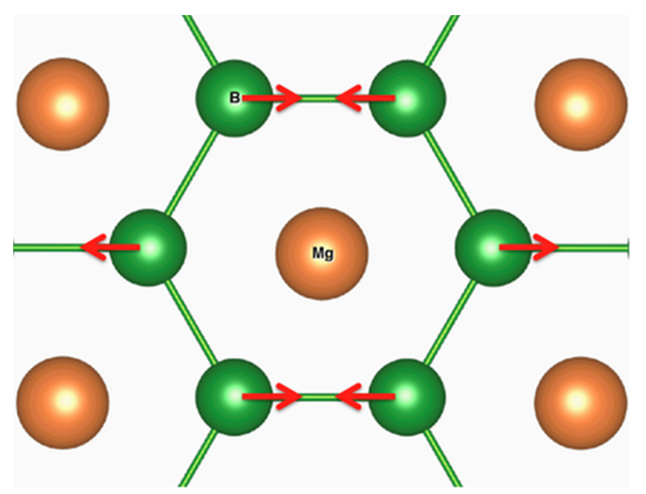

FIG. 3: Schematics of the polarization vector of one of the $E_{2 g}$ phonon modes in $\mathrm{MgB}_{2}$.

mation potential $D_{E_{2 g}}$ using the PBE functional is about $12.5 \mathrm{eV} / \AA$, which agrees well the result of An and Pickett [35]. The PBE0 functional, on the other hand, predicts a deformation potential of $26.3 \mathrm{eV} / \AA$ which nearly doubles that predicted by the PBE functional. This overestimated splitting between the two $p \sigma$ bands with the presence of the $E_{2 g}$ phonon distortion is consistent with the understanding that hybrid functionals tend to enhance the separation between occupied and unoccupied electronic states. Note that the PBE0 band structure is constructed from a self-consistent calculation on a $24 \times 24 \times 24$ uniform $k$-grid.

It should be mentioned that the strength of the elph coupling in $\mathrm{MgB}_{2}$ is so strong that the $E_{2 g}$ phonons in $\mathrm{MgB}_{2}$ are greatly renormalized as compared to the $E_{2 g}$ phonons in $\mathrm{AlB}_{2}[?]$. In fact, the $E_{2 g}$ phonons become so soft that the system appears to be close to a structural instability. The greatly exaggerated el-ph deformation potential calculated with hybrid functionals suggests that these functionals might actually predicts a structural instability for $\mathrm{MgB}_{2}$. To this end, we have cal-

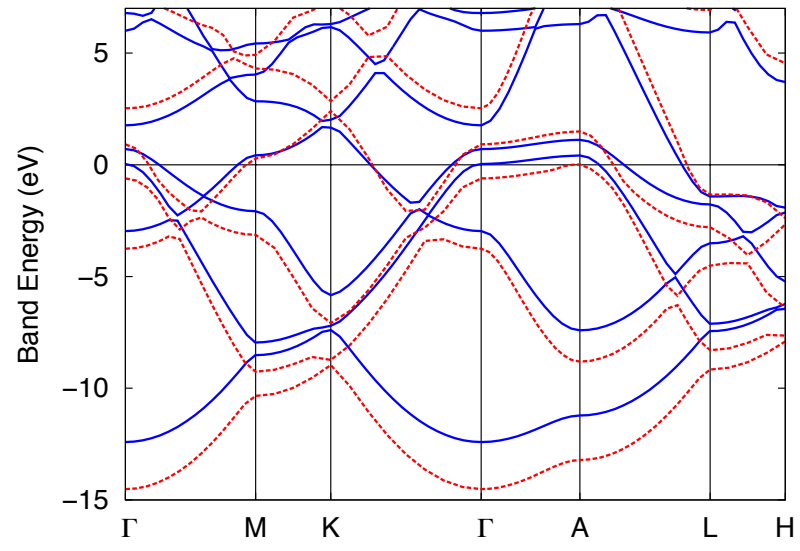

FIG. 4: Band structures of $\mathrm{MgB}_{2}$ calculated with a frozenphonon distortion along one of the $\mathrm{E}_{2 g}$ modes using the PBE (blue solid line) and PBE0 (red dashed line) functionals. The magnitude of the distortion is 0.05 a.u.

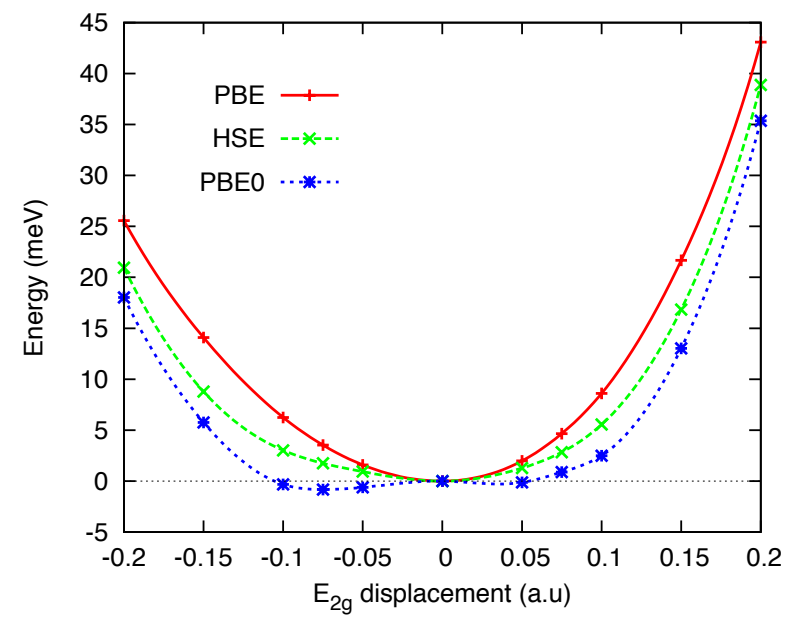

FIG. 5: Total energy of $\mathrm{MgB}_{2}$ as a function of the magnitude of a frozen-phonon distortion along one of the $\mathrm{E}_{2 g}$ phonon modes calculated using the PBE (red), HSE06 (green) and PBE0 (blue) functional.

culated the total energy of $\mathrm{MgB}_{2}$ as a function of the $E_{2 g}$ phonon displacement. As shown in Fig. 5, the potential curve calculated using the PBE functional (shown in red) shows a familiar third-order anharmonicity. The potential curve calculated using the HSE06 functional (green) suggests a greatly softened $E_{2 g}$ phonon compared with the PBE result (red), and the result from the PBE0 functional shows a double-well structure, indicating an instability against the $E_{2 g}$-like distortions. We emphasize that the LDA (or GGA) is able to describe well the phonon structure and el-ph coupling of this material. The HSE and PBE0 functionals definitely give much worse results compared with LDA (or GGA) functionals.

The combination of an inaccurate description of the electronic structure near the Fermi level and the wrong 
TABLE I: Calculated magnetic moments ( $\mu_{\mathrm{B}} /$ atom) and relative errors in comparison with experiment [40] for $\mathrm{Fe}$ and $\mathrm{Ni}$ using PBE and HSE06 functionals.

\begin{tabular}{ccc|cc|c}
\hline \hline & \multicolumn{2}{c|}{ PBE } & \multicolumn{2}{c|}{ HSE06 } & Experiment \\
& M & Error & M & Error & M \\
\hline $\mathrm{Fe}$ & 2.29 & $3 \%$ & 2.87 & $29 \%$ & 2.22 \\
$\mathrm{Ni}$ & 0.70 & $13 \%$ & 1.00 & $61 \%$ & 0.62 \\
\hline \hline
\end{tabular}

predictions of el-ph coupling and lattice stability suggests that hybrid functionals, especially the PBE0 functional, should probably never be used for metal systems, in particular, for the study of metallic properties that are sensitive to the electronic structure near the Fermi energy as we have discussed earlier. The inaccurate or false results for simple systems such as $\mathrm{Na}$ or $\mathrm{MgB}_{2}$ predicted by hybrid functionals, however, may not seem to be disturbing enough since these functionals are usually not used for simple systems. However, one can also argue that a reliable energy functional should work equally well for both simple and more complex (e.g., strongly correlated) systems.

Finally, we examine further into the limitations and applicability of hybrid functionals to the understanding of magnetic metals. One of the advantages of hybrid functionals (compared with LDA or GGA functionals) is that they improve the exchange energy functional and reduce the self-interaction error, which help to open the band gap for semiconductors. This advantage should be examined with great caution since it also produces erroneous predictions for a range of metallic properties as discussed above. Another consequence of including a fraction of the HF exchange energy (either in the original form such as that in the PBE0 functional or in the screened form as in the case of HSE functional) is that it enhances the localization of electronic states and the exchange splitting between the occupied (or majority spin) and unoccupied (or minority spin) states. This enhancement, however, does not guarantee a better description of magnetism.

To illustrate this issue, we have performed spinpolarized calculations for iron and nickel using the PBE and the HSE hybrid functionals. Table I compares the calculated magnetic moments of these two systems with experiments. The HSE06 functional overestimates the spin magnetic moment by about $29 \%$ and $61 \%$ for Fe and $\mathrm{Ni}$, respectively. On the other hand, the magnetism predicted by the PBE functional is in reasonable agreement with experiment [40]. We mention that similar problem has also been discussed recently [41, 42] that hybrid functionals predict wrong magnetic ground states for $\mathrm{Co}, \mathrm{Ni}$, $\mathrm{Ph}, \mathrm{Pd}$ and Pt.

\section{CONCLUSION}

In conclusion, we have shown that there are serious limitations of hybrid functionals in predicting fundamental properties of metals, especially for properties that are sensitive to (or dertermined by) electronic structure near the Fermi energy. These difficulties can be traced back to the overly simplified HF-like exchange energy included in hybrid functionals which is known to give an the inaccurate description of electronic structure near the Fermi level. Introducing a short-ranged Coulomb potential as in the case of the HSE functional does alleviate some of the problems, but major difficulties remain unchanged. Although computationally demanding, hybrid functionals are becoming increasingly popular with the implicit assumption that they will give a more faithful description of the underlying electronic structure. Our results raise a serious question regarding the applicability of hybrid functionals to the study metallic systems. We would also like to mention that hybrid functionals do not always fail for systems involving metals. Some integrated properties of metals (such as total energy, elastic properties, some structural properties), hybrid functionals may give similar or better results compared with LDA or GGA functionals.

\section{ACKNOWLEDGEMENT}

This work is supported by the National Science Foundation under Grant No. DMR-0946404 and DMR1506669. Y.S. and S.Z. are supported by the Department of Energy under Grant No. DE-SC0002623. We acknowledge the computational support provided by the Center for Computational Research at the University at Buffalo, SUNY.

[1] P. Hohenberg and W. Kohn, Phys. Rev. 136, B864 (1964).

[2] W. Kohn and L. J. Sham, Phys. Rev. 140, A1133 (1965).

[3] D. M. Ceperley and B. J. Alder, Phys. Rev. Lett. 45, 566 (1980).

[4] D. C. Langreth and M. J. Mehl, Phys. Rev. B 28, 1809 (1983).

[5] A. D. Becke, Phys. Rev. A 38, 3098 (1988).

[6] J. P. Perdew, J. A. Chevary, S. H. Vosko, K. A. Jackson, M. R. Pederson, D. J. Singh, and C. Fiolhais, Phys. Rev. B 46, 6671 (1992).

[7] A. D. Becke, The Journal of Chemical Physics, 98, 1372 (1993).

[8] M. Ernzerhof and G. E. Scuseria, The Journal of Chemical Physics 110, 5029 (1999).

[9] C. Adamo and V. Barone, The Journal of Chemical Physics 110, 6158 (1999). 
[10] J. Muscat, A. Wander, and N. Harrison, Chemical Physics Letters 342, 397 (2001).

[11] J. Heyd, J. E. Peralta, G. E. Scuseria, and R. L. Martin, The Journal of Chemical Physics 123, 174101 (2005).

[12] J. Paier, M. Marsman, K. Hummer, G. Kresse, I. C. Gerber, and J. G. ngyn, The Journal of Chemical Physics 124, 154709 (2006).

[13] J. L. F. Da Silva, M. V. Ganduglia-Pirovano, J. Sauer, V. Bayer, and G. Kresse, Phys. Rev. B 75, 045121 (2007).

[14] M. Betzinger, C. Friedrich, and S. Blugel, Phys. Rev. B 81, 195117 (2010).

[15] F. Iori, M. Gatti, and A. Rubio, Phys. Rev. B 85, 115129 (2012).

[16] R. Murphy and D. R. Strongin, Surface Science Reports 64, 1 (2009).

[17] A. Ennaoui, S. Fiechter, C. Pettenkofer, N. AlonsoVante, K. Bker, M. Bronold, C. Hpfner, and H. Tributsch, Solar Energy Materials and Solar Cells 29, 289 (1993).

[18] J. Heyd, G. E. Scuseria, and M. Ernzerhof, J. Chem. Phys. 118, 8207 (2003).

[19] A. V. Krukau, O. A. Vydrov, A. F. Izmaylov, and G. E. Scuseria, The Journal of Chemical Physics 125, 224106 (2006).

[20] R. Sun, M. K. Y. Chan, and G. Ceder, Phys. Rev. B 83 , $235311(2011)$

[21] J. Heyd and G. E. Scuseria, The Journal of Chemical Physics 121 (2004).

[22] M. Marsman, J. Paier, A. Stroppa, and G. Kresse, Journal of Physics: Condensed Matter 20, 064201 (2008).

[23] L. Schimka, J. Harl, A. Stroppa, A. Grneis, M. Marsman, F. Mittendorfer, and G. Kresse, Nature Materials 9, 741 (2010).

[24] O. T. Hofmann, V. Atalla, N. Moll, P. Rinke, and M. Scheffler, New Journal of Physics 15, 123028 (2013).

[25] P. Giannozzi, S. Baroni, N. Bonini, M. Calandra, R. Car, C. Cavazzoni, D. Ceresoli, G. L. Chiarotti, M. Cococcioni, I. Dabo, et al., J. of Phys.: Conden. Matt. 21, 395502 (2009).
[26] J. P. Perdew, K. Burke, and M. Ernzerhof, Phys. Rev. Lett. 77, 3865 (1996)

[27] J. P. Perdew, M. Ernzerhof, and K. Burke, J. Chem. Phys. 105, 9982 (1996).

[28] N. Troullier and J. L. Martins, Phys. Rev. B 43, 1993 (1991).

[29] E. Jensen and E. W. Plummer, Phys. Rev. Lett. 55, 1912 (1985).

[30] I.-W. Lyo and E. W. Plummer, Phys. Rev. Lett. 60, 1558 (1988).

[31] J. E. Northrup, M. S. Hybertsen, and S. G. Louie, Phys. Rev. B 39, 8198 (1989).

[32] N. Ashcroft and N. Mermin, Solid State Physics (Saunders College, Philadelphia, 1976).

[33] J. Nagamatsu, N. Nakagawa, T. Muranaka, Y. Zenitani, and J. Akimitsu, Nature 410, 63 (2001).

[34] S. L. Bud'ko, G. Lapertot, C. Petrovic, C. E. Cunningham, N. Anderson, and P. C. Canfield, Phys. Rev. Lett. 86, 1877 (2001)

[35] J. M. An and W. E. Pickett, Phys. Rev. Lett. 86, 4366 (2001).

[36] A. Y. Liu, I. I. Mazin, and J. Kortus, Phys. Rev. Lett. 87, 087005 (2001)

[37] H. J. Choi, D. Roundy, H. Sun, M. L. Cohen, and S. G. Louie, Phys. Rev. B 66, 020513 (2002).

[38] T. Yildirim, O. Gülseren, J. W. Lynn, C. M. Brown, T. J. Udovic, Q. Huang, N. Rogado, K. A. Regan, M. A. Hayward, J. S. Slusky, et al., Phys. Rev. Lett. 87, 037001 (2001).

[39] P. Zhang, S. G. Louie, and M. L. Cohen, Phys. Rev. Lett. 94, 225502 (2005).

[40] J. Crangle and G. M. Goodman, Proceedings of the Royal Society of London. Series A, Mathematical and Physical Sciences 321, pp. 477 (1971).

[41] F. Tran, D. Koller, and P. Blaha, Phys. Rev. B 86, 134406 (2012).

[42] Y.-R. Jang and B. D. Yu, Journal of the Physical Society of Japan 81, 114715 (2012). 